\title{
Cannabinoid Hyperemesis Syndrome: A Case Report and Review of Pathophysiology
}

\author{
Corina L. lacopetti, BS and Clifford D. Packer, MD
}

Cannabis is the most widely used illicit drug in the United States, with lifetime prevalence of use estimated at $42 \%$ to $46 \%$. The antiemetic properties of cannabis are well-known by the medical community and the general public; however, less well-recognized is the paradoxical potential for certain chronic users to develop hyperemesis. We describe in this case a patient with prior extensive work-up for nausea and vomiting and previous diagnosis of cyclic vomiting syndrome who presented with characteristic features of cannabinoid hyperemesis syndrome. We review the current literature for this condition and highlight potential mechanisms for its pathogenesis.

Keywords: Cannabis; Cyclic vomiting; Hyperemesis; Marijuana

Corresponding Author:

Corina L. lacopetti, BS

Case Western University School of Medicine

Office of Student Affairs, Robbins Bdg, E-42।

10900 Euclid Ave

Cleveland, OH 44106 USA

Email: cli3@case.edu

Received: May 21, 2013

Revised: August I, 2013

Accepted: August 7, 2013

doi: $10.3121 / \mathrm{cmr} .2013 .1179$
A disorder, hypertension, and gastroesophageal reflux presented to a tertiary care center with a two-day history of severe nausea and intractable vomiting. The vomiting began with no identifiable precipitating factor, was bilious but nonbloody, and occurred as frequently as six times per hour. The patient reported abdominal pain and lightheadedness secondary to dehydration but denied any fever, coryza, change in bowel movements, or sick contacts.

The patient had been experiencing similar episodes recurrently for the past 2 years, frequently associated with life stressors and requiring five previous hospitalizations. He had received an extensive work-up, including laboratory tests, kidney-ureter-bladder radiograph, computed tomography of the abdomen, abdominal ultrasound, gastric emptying study, and esophagogastroduodenoscopy. All tests returned negative for identifiable pathology, with the exception of electrolyte disturbances secondary to protracted emesis. As a result, the patient was diagnosed with cyclic vomiting syndrome.

Notably, however, the patient had been an intermittent, recreational marijuana user for many years prior but had escalated to daily use over the past 2 years for symptom relief, citing the known antiemetic effects of cannabis. In addition, he described a compulsive pattern of bathing, stating that he often felt nauseated when having bowel movements and had discovered that transitioning directly from the toilet to a hot shower helped resolve his nausea. He described turning the shower temperature to a maximum, noting that it felt as though the hot water washed away his nausea. The patient often required several such showers per day and had been doing this for at least one year.

His home medications included only citalopram and omeprazole. Family history was non-contributory. The patient drank two beers daily and had a history of 
tobacco use in the past. His military history was significant for two tours to Iraq as an Army paratrooper. On examination, his vitals were temperature $98.2^{\circ} \mathrm{F}$, heart rate $87 \mathrm{bpm}$, blood pressure 154/95 $\mathrm{mmHg}$, and respiratory rate $20 \mathrm{bpm}$. Blood oxygen saturation was $98 \%$ on room air. Physical examination was benign, with soft, non-tender, non-distended abdomen. Laboratory studies demonstrated leukocytosis $\left(19.6 \mathrm{~K} / \mathrm{cm}^{2}\right)$, hyponatremia $(133 \mathrm{mEq} / \mathrm{L})$, hypokalemia $(3.3 \mathrm{mEq} / \mathrm{L})$, and hypochloremia $(96 \mathrm{mmol} / \mathrm{L})$ with normal liver function tests, all consistent with imbalances due to hyperemesis. The diagnosis was revised to cannabinoid hyperemesis syndrome, and the patient was treated with intravenous fluids, intravenous ondansetron, and oral omeprazole. Unfortunately, despite counseling, the patient was not amenable to cannabis cessation at that time. He was discharged home in stable condition, and advised to follow up with his primary care physician.

\section{Discussion}

Cannabis is the most widely used illicit drug in the United States, with lifetime prevalence of use estimated at $42 \%$ to $46 \% .{ }^{1}$ Cannabinoid hyperemesis is a syndrome seen in some chronic cannabis users. It is characterized by cyclic nausea and vomiting, as well as a pattern of compulsive hot water bathing for symptom relief. Symptoms completely resolve with cannabis cessation. This clinical entity was first noted by J.H. Allen in South Australia in 2001, with a follow-up case series of nine patients published in 2004. ${ }^{2}$ Since then, several case reports and case series have appeared in the literature.

Based on the largest case series to date, which examined 98 patients, authors at the Mayo Clinic have proposed clinical criteria for cannabinoid hyperemesis syndrome (CHS). Essential for diagnosis is long-term cannabis use, though no specific definition of "long-term" has been established. Major features for the syndrome include severe cyclic nausea and vomiting, resolution of symptoms with cannabis cessation, symptom relief with hot showers or baths, abdominal pain (epigastric or periumbilical), and at least weekly marijuana use. Supportive features include age less than 50 years, weight loss of greater than five kilograms, morning predominance of symptoms, normal bowel habits, and negative laboratory, radiographic, and endoscopic test results. In addition to these features, it has also been noted that many patients report autonomic symptoms such as flushing and diaphoresis. $^{3}$

The compulsive bathing seen in CHS is a pattern not noted in other vomiting disorders and is, thus, hypothesized to be a pathognomonic feature. Symptom relief from bathing appears to be temperature-dependent, with hotter water yielding more effective relief. This bathing behavior is not considered psychotic or obsessive-compulsive, but is instead learned. Often absent during initial episodes of nausea and vomiting, it rapidly becomes a compulsion once symptom relief is established, disappears during intervening periods of normal health, and resolves fully with cessation of cannabis use. ${ }^{2}$ Notably, cyclic vomiting due to CHS has been suggested to be refractory to common anti-emetics; thus, hot water bathing is often the only alleviating factor patients can recognize.

Patients with CHS generally have a history of multiple emergency department visits and hospitalizations. They often receive extensive work-ups, including diagnostic imaging and invasive procedures, and are often diagnosed with cyclic vomiting syndrome (CVS) when no clear evidence for pathology results from the evaluation. CVS has an unknown etiology, though it is associated with psychological stressors and family history of migraines. Significantly, compulsive bathing patterns are not observed with this entity and seem to be distinctive to $\mathrm{CHS}^{4}$

For this reason, once healthcare providers do not suspect lifethreatening etiologies for a patient's hyperemesis, it is suggested that they inquire if the patient's symptoms improve with hot showers or baths. In addition, inquiries into cannabis use, including frequency and duration, are helpful in assessing the likelihood that the patient may be presenting with CHS. As Wallace et $\mathrm{al}^{4}$ suggest, if the patient endorses compulsive hot water bathing behaviors but denies cannabis use, a urine drug screen is warranted, as these behaviors may be considered pathognomonic for CHS. In pursuing these lines of evaluation, the patient and provider can come to the appropriate diagnosis more quickly, with reduction in patient morbidity and use of health care resources. Treatment for CHS is supportive, with cannabis cessation and fluid replacement if clinically indicated. ${ }^{4}$

Various theories have been proposed to explain the pathophysiology of CHS, employing both the pharmacokinetics and pharmacodynamics of cannabinoids. First, delta 9-tetrahydrocannabinol (THC), the main active compound in cannabis, has a tendency to sequester in fat with slow rediffusion back into the serum. This tendency seems to create different phases of excretion with the rate of elimination slowing in each successive phase: a half-life of approximately 2 hours has been measured in the first 8 hours after administration, but a half-life of up to 12.6 days has been measured when the observation period is extended to 4 weeks. ${ }^{6}$ Thus, THC's large volume of distribution may lead to accumulation in chronic users and potential toxicity in predisposed patients. ${ }^{5}$

Both endogenous and exogenous cannabinoids act on G-protein coupled receptors $\mathrm{CB}_{1}$ and $\mathrm{CB}_{2}$, with $\mathrm{CB}_{1}$ receptors in the CNS and GI tract being implicated in most of cannabis's known effects. THC activates $\mathrm{CB}_{1}$ receptors in the enteric nervous system to decrease gastric motility, which would incline toward nausea and vomiting. However, THC also exerts a variety of antiemetic effects centrally at the brainstem dorsal vagal complex: it activates $\mathrm{CB}_{1}$ receptors, ${ }^{5}$ noncompetitively inhibits $5-\mathrm{HT}_{3}$ serotonin receptors (the target of $5-\mathrm{HT}_{3}$ antagonists such as ondansetron), and potentially also inhibits serotonin release via pre-synaptic $\mathrm{CB}_{1}$ receptors. $^{7}$ This central action is postulated to outweigh 
THC's pro-emetic effect on the GI tract since cannabis use is not traditionally associated with nausea and vomiting. ${ }^{5}$

Given this extensive central antiemetic action, it is difficult to determine how some chronic cannabis users develop CHS. Some authors have suggested that THC's pro-emetic effect in the gut outweighs its central antiemetic effect in susceptible individuals. ${ }^{5}$ However, of the 61 patients in the Mayo Clinic case series who received gastric scintigraphy, $46 \%$ had normal gastric emptying, $30 \%$ had delayed emptying, and $25 \%$ had rapid emptying. ${ }^{3}$ Impaired gastric motility, thus, does not appear to be a consistent factor in patients with CHS. Other authors have suggested that different compounds in cannabis, namely cannabidiol and cannabigerol, may mediate the pathogenesis of CHS. ${ }^{5}$ Interestingly, cannabidiol, the principal non-psychoactive component of marijuana, has been found to have a biphasic effect on vomiting in shrews. In both cis-platinum and lithium-treated shrews, low doses of cannabidiol suppressed vomiting, but high doses potentiated vomiting. In contrast, THC had a dose-dependent antiemetic effect in both groups of shrews. ${ }^{8,9}$ This suggests that high levels of cannabidiol might play a role in the development of CHS in very heavy marijuana users.

Finally, though the pathophysiology of the compulsive bathing distinctive to CHS is not yet well-understood, the prevailing theory suggests that cannabis toxicity may lead to impaired thermoregulation, with hot water bathing acting to relieve decreases in core body temperature. ${ }^{3}$ Indeed, in rodents the hypothermic effect of THC is well-established. Acting via hypothalamic $\mathrm{CB}_{1}$ receptors, THC induces dosedependent hypothermia that is reversible upon administration of $\mathrm{CB}_{1}$ antagonists. ${ }^{9}{ }^{10}$ Interestingly, the SSRI fluoxetine has been shown to modulate this action by attenuating hypothermia when fluoxetine administration precedes that of THC, but potentiating hypothermia when fluoxetine follows THC. ${ }^{11}$ This finding is particularly intriguing, as our patient was also regularly taking an SSRI, citalopram. Given the interaction of cannabinoids with the serotonergic system, and given the frequent concomitance of substance use and mood disorders (which can both disrupt the serotonergic system independently and lead to administration of psychotropic medications), one might hypothesize that derangements in the serotonergic system could predispose to development of CHS. For instance, the inhibitory effects of THC on serotonin release and its concurrent $5-\mathrm{HT}_{3}$ receptor blockade could lead to upregulation of $5-\mathrm{HT}_{3}$ receptors in susceptible chronic users, perhaps causing paradoxically increased sensitivity to emetogenic stimuli, either with administration of medications that increase serotonin availability or with temporary cessation of cannabis use.

\section{Conclusion}

Clinicians should be aware that cannabis can have paradoxically emetogenic effects in some habitual users and that the pathognomonic features of cannabinoid hyperemesis syndrome are recurrent bouts of vomiting and symptom relief with hot water bathing. Increased awareness of CHS could reduce the expense and potential morbidity of extensive diagnostic work-ups for these patients. Finally, as the majority of marijuana users do not seem to develop CHS, further studies are needed to understand the risk factors and pathogenesis of this syndrome.

\section{References}

1. Degenhardt L, Chiu WT, Sampson N, Kessler RC, Anthony JC, Angermeyer M, Bruffaerts R, de Girolamo G, Gureje O, Huang Y, Karam A, Kostyuchenko S, Lepine JP, Mora ME, Neumark Y, Ormel JH, Pinto-Meza A, Posada-Villa J, Stein DJ, Takeshima T, Wells JE. Toward a global view of alcohol, tobacco, cannabis, and cocaine use: findings from the WHO World Mental Health Surveys. PLoS Med 2008;5:e141.

2. Allen JH, de Moore GM, Heddle R, Twartz JC. Cannabinoid hyperemesis: cyclical hyperemesis in association with chronic cannabis abuse. Gut 2004;53:1566-1570.

3. Simonetto DA, Oxentenko AS, Herman ML, Szostek JH. Cannabinoid hyperemesis: a case series of 98 patients. Mayo Clin Proc 2012;87:114-119.

4. Wallace EA, Andrews SE, Garmany CL, Jelley MJ. Cannabinoid hyperemesis syndrome: literature review and proposed diagnosis and treatment algorithm. South Med J. 2011;104:659-664.

5. Galli, JA, Sawaya RA, Friedenberg FK. Cannabinoid hyperemesis syndrome. Curr Drug Abuse Rev 2011;4: 241-249.

6. Toennes SW, Ramaekers JG, Theunissen EL, Moeller MR, Kauert GF. Comparison of cannabinoid pharmacokinetic properties in occasional and heavy user smoking a marijuana or placebo joint. J Anal Toxicol 2008;32:470-477.

7. Parker LA, Rock EM, Limebeer CL. Regulation of nausea and vomiting by cannabinoids. Br J Pharmacol 2011;163: $1411-1422$.

8. Kwiatkowska M, Parker LA, Burton P, Mechoulam R. A comparative analysis of the potential of cannabinoids to suppress cisplatin-induced emesis in the Suncus murinus (house musk shrew). Psychopharmacology (Berl) 2004; 174:254-259.

9. Parker LA, Kwiatkowska M, Burton P, Mechoulam R. Effect of cannabinoids on lithium-induced vomiting in the Sincus murinus (house musk shrew). Psychopharmacol (Berl) 2004;171:156-161.

10. Varvel SA, Bridgen DT, Tao Q, Thomas BF, Martin BR, Lichtman AH. Delta9-tetrahydrocannbinol accounts for the antinociceptive, hypothermic, and cataleptic effects of marijuana in mice. J Pharmacol Exp Ther 2005;314:329-337.

11. Smirnov MS, Kiyatkin EA. Behavioral and temperature effects of delta 9-tetrahydrocannabinol in human-relevant doses in rats. Brain Res 2008;1228:145-160.

12. Malone DT, Taylor DA. Modulation of delta9tetrahydrocannabinol-induced hypothermia by fluoxetine in the rat. Br J Pharmacol 1998;124:1419-1424.

\section{Author Affiliations}

Corina L. Iacopetti, BS $S^{*}$ and Clifford D. Packer, $M D^{*+}$

${ }^{*}$ Case Western Reserve University School of Medicine, Cleveland, $\mathrm{OH}$ 44106, USA

'Louis Stokes Cleveland Veterans Affairs Medical Center, Cleveland, $\mathrm{OH} 44106$, USA 